\title{
Transcriptional profiling of oral squamous cell carcinoma using formalin- fixed paraffin-embedded samples
}

Article type: Article

Abstract:

Despite the advances in cancer treatment, the 5-year survival rate for oral cancer has not changed significantly for the past 40 years and still remains among the worst of all anatomic sites. Gene expression microarrays have been used successfully in the identification of genetic alterations in cancer development, however, these have hitherto been limited by the need for specimens with good quality intact RNA. Here, we demonstrated the use of formalin-fixed paraffin-embedded tissues in microarray experiments to identify genes differentially expressed between cancerous and normal oral tissues. Forty-three tissue samples were macrodissected and gene expression analyses were conducted using the Illumina DASL assay. We report RNA yield of 2.4 and $0.8 \mu \mathrm{g} / \mathrm{mm} 3$ from tumour and normal tissues, respectively and this correlated directly with the tissue volume used for RNA extraction. Using unsupervised hierarchical clustering, distinct gene expression profiles for tumour and normal samples could be generated, and differentially expressed genes could be identified. The majority of these genes were involved in regulation of apoptosis and cell cycle, metastasis and cell adhesion including BCL2A1, BIRC5, MMP1, MMP9 and ITGB4. Representative genes were further validated in independent samples suggesting that these genes may be directly associated with oral cancer development. The ability to conduct microarrays on formalin-fixed paraffin-embedded specimens represents a significant advancement that could open up avenues for finding genes that could be used as prognostication and predictive tools for cancer.

\begin{tabular}{|c|l|}
\hline Author & $\begin{array}{l}\text { Saleh, A. ; Zain, R. B. ; Hussaini, H. ; Ng, F. ; Tanavde, V. ; } \\
\text { Hamid, S. ; Chow, A. T. ; Lim, G. S. ; Abraham, M. T. ; Teo, S. H. } \\
\text { Cheong, S. C. }\end{array}$ \\
\hline Source & Oral Oncology \\
\hline ISSN & $1368-8375$ \\
\hline DOI & $10.1016 /$ j.oraloncology.2010.02.022 \\
\hline Volume & 46 \\
\hline Page & $379-386$ \\
\hline Year & 2010 \\
\hline
\end{tabular}

Keyword:

polymerase chain-reaction; gene-expression profiles; breast-cancer; molecular classification; capture microdissection; microarray analysis; messenger-rna; bcl-2 homolog; neck-cancer; head 
Please Cite As:

SALEH, A., ZAIN, R. B., HUSSAINI, H., NG, F., TANAVDE, V., HAMID, S., CHOW, A. T., LIM, G. S., ABRAHAM, M. T., TEO, S. H. \& CHEONG, S. C. 2010. Transcriptional profiling of oral squamous cell carcinoma using formalinfixed paraffin-embedded samples. Oral Oncology, 46, 379-386.

URL:

- http://www.scopus.com/inward/record.url?eid=2-s2.077950925055\&partnerlD=40\&md5=c2e3ba95d0f1ca1 d7336966b81b103f8

- http://cat.inist.fr/?aModele=afficheN\&cpsidt=22730595

- http://www.sciencedirect.com/science/article/pii/S1368837510000692

- http://www.ncbi.nlm.nih.gov/pubmed/20371203

- http://www.oraloncology.com/article/S1368-8375(10)00069-2/abstract

- http://apps.webofknowledge.com/full record.do?product=WOS\&search mode=M arkedList\&qid=58\&SID=3BbLpnGmFI374ee9@Jc\&page =1\&doc=2\&colName=W $\underline{\mathrm{OS}}$ 